\title{
Contextual Visualization of Ontological Models
}

\author{
Jozef Vrana \\ Department of Cybernetics and Artificial Intelligence \\ Faculty of Electrical Engineering and Informatics \\ Technical University in Kosice \\ Letna 9, 04200 Kosice, Slovakia \\ jozef.Vrana@tuke.sk
}

\begin{abstract}
In this paper we summarize the rationale, motivation, and functionality of our method for visualizing ontologies based on the notion of ontology summaries and key concepts that are most likely to describe the topical focus of a given ontology. This approach offers an alternative to the usual top-down browsing of extensive ontological trees, and our preliminary studies show that people are more likely to comprehend the topicality of a given ontology from the key concepts than they would be from the top-level classes. Inspiration for the approach mostly came from natural human approach to tackle problems at different levels of analysis. These different levels of analysis are instantiated here in the form of (i) calculating conceptual summaries of loaded ontologies, (ii) enriching these with topological summaries, and (iii) providing a selection of interactive means to get a quick snapshot of what the ontology in question is about.
\end{abstract}

\section{Categories and Subject Descriptors}

I.2.6 [Artificial Intelligence]: Learning-Knowledge acquisition; H.3 [Information Storage and Retrieval]: On-line Information Services-Web-based services; I.2.4 [Artificial Intelligence]: Knowledge Representation Formalisms and Methods-Representation languages, Semantic networks

\section{Keywords}

ontology, ontology visualization, ontology summaries, key concept

\footnotetext{
*Recommended by thesis supervisor:

Assoc. Prof. Marian Mach

Defended at Faculty of Electrical Engineering and Informatics, Technical University in Kosice on September 29, 2010.

(C) Copyright 2010. All rights reserved. Permission to make digital or hard copies of part or all of this work for personal or classroom use is granted without fee provided that copies are not made or distributed for profit or commercial advantage and that copies show this notice on the first page or initial screen of a display along with the full citation. Copyrights for components of this work owned by others than ACM must be honored. Abstracting with credit is permitted. To copy otherwise, to republish, to post on servers, to redistribute to lists, or to use any component of this work in other works requires prior specific permission and/or a fee. Permissions may be requested from STU Press, Vazovova 5, 81107 Bratislava, Slovakia.

Vrana, J. Contextual Visualization of Ontological Models. Information Sciences and Technologies Bulletin of the ACM Slovakia, Vol. 2, No. 2 (2010) 130-136
}

\section{Introduction}

Many past projects developing semantic tools paid only limited attention to users - with the result that ontology engineering technology is tried and discarded by users after a brief trial. Needs of non-experts has been neglected so far which resulted in unwillingness to accept ontology as a useful and formally correct resource of information. The major reason for existing ontology tools being discarded by users is that they are simply too difficult to use. Among the functionalities that capture the eye of most users, many existing tools provide reasonable editing facilities and some visualization/navigation support. However, as proven in two large scale studies of main ontology engineering environments [4], the user support is far from ideal. Among many identified issues, several of them are considered major from our perspective:

- studies revealed that complex graphs and lenghtly trees starting at the abstract levels as primary visual elements are generally found to be a poor metaphor for user needs, and only tended to add the already steep learning curve for a non-expert user.

- while Protege users surveyed about their experience with visualization, they often claimed that such tools are too complex and do not reflect users' models of what they would normally want to see in unfamiliar ontologies.

- we also discovered that non-experts often find themselves less efficient due to the lack of simple visualization and navigation support especially in terms of obtaining overviews of provided ontologies.

- generally, visual interaction was probably the area where participants had strongest reactions, commenting on confusing presentation that leads to a plodding search across the whole collection of entities in an ontology.

The above section of observations from the previous user studies motivated us to propose an idea of visualization and visual interaction based on ontology summaries. The reason why the summaries caught our attention is simple - with a number of tools allowing users to access and reuse ontological resources, it is more likely a user comes across ontologies, which s/he needs to make sense of, to quickly preview them and decide whether they are worth downloading. Obviously, it is impossible to make a decision based on ontology URI that has no descriptive value, nor the whole content of ontology which provides usually 
too much information to be digested. Here is where summaries come to provide shortened presentation, highlighting the most important features of an evaluated ontology. As a result, the three key pillars were defined in order to tackle all mentioned issues:

1. Focus on non-experts who deals with ontologies without having deep insight of formalism or logic and encourage them to take advantages of such an approach in the same way as users works with the Internet without having understanding of related technologies.

2. Neglecting the ontologies re-use: Even though majority of ontologies are developed to suit particular problem or certain type of task, the main benefit of this technology is the possibility of re-use for future applications. When building a new ontology it is better to do it by re-using existing ontologies rather than developing everything from scratch. Ontology re-use, which is one of basic paradigms of knowledge modelling, is a challenging problem due to lack of existing techniques and tools.

\section{Key principles}

In this section of the paper we present key steps on the way from ontology to its meaningful snapshot by facilitating the process of ontology understanding. While details of this transition can by found in [8], a brief explanation of key principles of our approach will be provided.

Vector description of the domain Vector description is the most essential step that aims to summarize information about a domain which is being described. It stores relevant information into a vector of weighted terms where weight reflects importance of a particular term regarding the domain. The domain is therefore represented by one vector that may be used as a reference entity for comparison.

Key concepts acquisition When deciding on importance of particular parts of ontology and searching for concepts with the highest information value it is vital to be able to rank concepts according to their importance. The notion of key concepts is based on topological as well as cognitive measures and was developed on Knowledge Media institute. In terms of reducing information overload, key concepts are used as a representation of all concepts in ontology.

Vector description of the key concepts Vector that contains the description of a concept regarding its ontology and it is built using related (neighbour) concepts to cover the context of the described concept. Thus, vector description is created for each key concept from ontology and all these vectors together yields definition of an ontology.

Similarity measures All former steps give a setting where two kinds of vector descriptions are available (vectors of domains; vectors of the ontology) and then each key concept vector can be compared with all domain vectors to quantify similarities. The highest value across the whole collection of similarities determines the best match between key concepts and domain.
Visualisation in context In spite of key concepts being fairly solid approximation of an ontology, they are ambiguous and on their own are hardly enough to conceive the meaning. Here is where the geographical maps come to help us better explain the meaning of key concepts based on context. In this metaphor the domains act as a background of a map, defining area of interest or setting of reference. Key concepts representing points are distributed on the map . Together they create a comprehensible and sensible way of explaining key concepts (and consequently also an ontology) to non-experts.

Navigation in ontology All existing tools for visualisation of ontologies [5] are provided with navigation functionality as ontologies are often too complex to be depicted altogether. Facilitation of navigation is without a doubt essential and helps a user to better define his requirements.

The process of ontology re-use support is laid above, particularly in terms of determining suitability of particular ontology from predefined criterion. Forthcoming sections discuss this process in deeper granularity.

\section{Vector description of the domain}

Ontology concept is much more than just a label that is used to refer to the concept in natural language. Typically such a concept is set in certain kind of environment ${ }^{1}$ which further extends its meaning and makes it a formal, unambiguous description of the object. Many existing visualization techniques depict ontology objects solely as terms regardless their context or environment. On the other hand, though, there is a strong demands for comprehensible and brief view presented by a visualization tool, missing out inconspicuous information about the ontology.

However contradicted this specification appears to be, it can be fulfilled with intelligent data filtering and analysis of data significance. We tackled those requirements by using vector space model, an algebraic model for representing text documents (or any other object) as vectors of identifiers [7]. The vector space model is well established within information retrieval and commonly used for representing text documents.

$$
d_{i}=\left(w_{i 1}, \ldots, w_{i M}\right)
$$

The equation 1 . defines a vector, where $w_{i k}$ is weight of term $t_{i k}$ in document $d_{i}$. By applying the vector space model with the domain of interest in the role of a document, we end up with exactly the same formula representing importance of terms regarding particular domain. The domain represents a reference entity against which the ontology is compared. Thus it is, in some extent, a golden standard but unlike the traditional golden standard, this one is generated automatically.

By creating so-called vector description of the domain, that consists of terms which fit into a particular domain

\footnotetext{
${ }^{1}$ other related concepts along with their relations and properties
} 


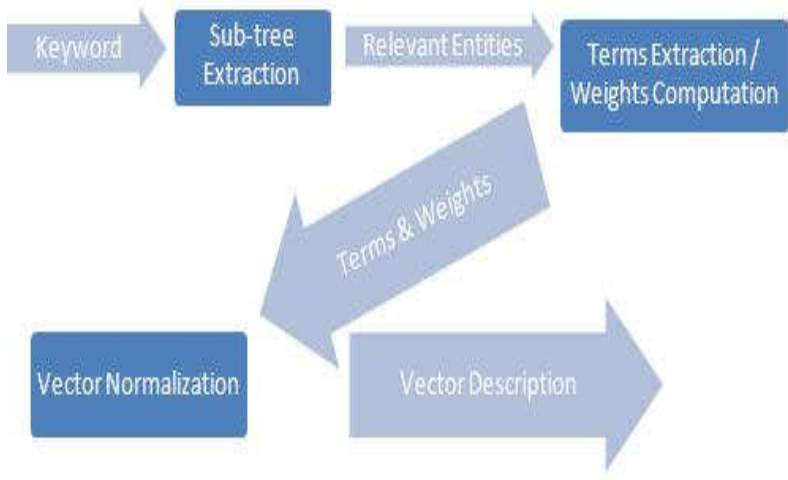

Figure 1: Scheme of generating the vector description of a domain shows actual steps of processing the input keyword alongside information flows between these steps.

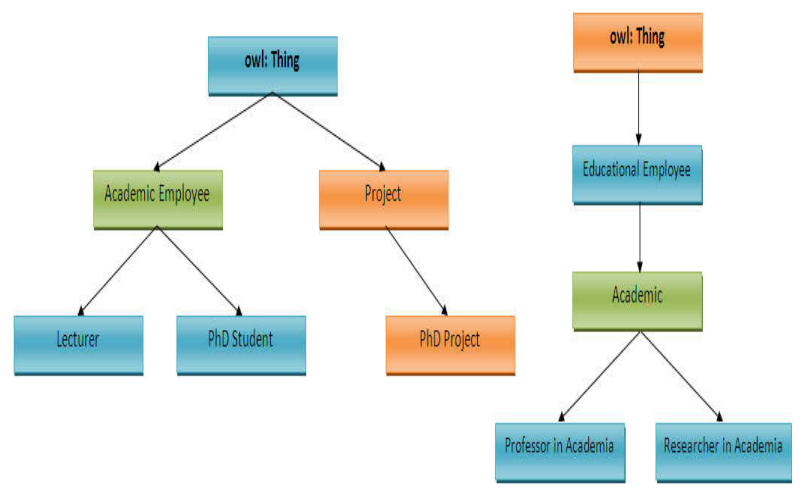

Figure 2: Graphs of two ontologies, where green nodes refers to original concepts whilst blue ones are their nearest surroundings.

with various degrees (represented by weights), we obtain a fairly solid approximation of the domain. The idea was to gather terms which are relevant to the domain in order to increase precision while comparing to an ontology. Such a representation not only captures real circumstances but it is as precise as all ontologies concerning the domain (included in the used ontology corpus).

At the beginning of building the vector representing a domain is a keyword ${ }^{2}$, a label of the domain serving as a root around which all related terms are grouped. The search engines (e.g., [1], [3]) are keyword based and so they can find ontologies where a specified keyword has emerged in the name of classes, instances or other concepts. When a concept containing the keyword is found in an ontology, we tend to explore the nearest neighbours (sub/super related concepts) e.g., isA link or any other bound in/from the original concept.

An example of two relevant ontologies is on Fig.2, where the keyword is in green block (Academic Employee is the original concept ${ }^{3}$ ) and neighbour concepts are in blue blocks ( for the graph on the left: owl:Thing; Lecturer; $\mathrm{PhD}$ Student). Only direct neighbours are taken into con-

\footnotetext{
${ }^{2}$ It also may be several keywords to describe one domain if necessary.

${ }^{3}$ Concept in which the keyword has occurred.
}

sideration as they represent the closest context of the original concept. All other concepts in ontology (on the picture in orange) have no bond with original concept and so they are left out from further calculations. The exact match between concept name and the user specified keyword is not required and therefore even concept Academic is considered original.

Ontology comprises a range of information though only labels and local names of concepts are found interesting for our purposes. The word concept includes classes, properties and individuals.

Afterwards, the obtained sub graph of ontology is being processed and decomposed into terms. In order not to lose data carried by links, these are reflected into initial weights. As depicted on the Fig.2, surrounding concepts extend the definition of the original concept found in the ontology ${ }^{4}$. At first, initial weights are established for original concept Academic Employee:

- for a Class the initial weight is $w_{O}=10+G$;

- for an Individual it is $w_{O}=G$;

- otherwise: $w_{O}=1$.

Weight for contextually-bounded is derived taking in account two measures, first of which is the initial weight of the original object and the second is the number of terms in its label ${ }^{5}$ as follows:

- Super object i.e. owl:Thing:

$$
w=\frac{w_{O}+G}{l}
$$

- Sub object i.e. Lecturer, PhD Student:

$$
w=\frac{w_{O}}{l}
$$

The label of each object typically consists of one or more words (e.g. Musical Instrument). In the formula $l$ stands for the number of terms in the label/local name. The constant $G$ that emerges in almost every formula is a value that reflects usersï£i requirements in terms of generality. In other words: user can prefer more general objects to less general objects. Thus, user is given the opportunity to express the preference by a number on the scale from 1 to 10 (1=least general; $10=$ very general). The described calculation is performed on every ontology, where the keyword was found. Several partial vectors (each for one ontology) are then aggregated into one final vector of the domain. The initial weight for certain term reflects its importance regarding certain ontology, in case we want to extend it on the whole ontology corpus, weights must be aggregated for each occurrence of the term in all partial vectors. At this point the initial weights are being aggregated in case one term is present more than once in partial vectors according to formula:

${ }^{4}$ All calculations are performed only on the left side graph to keep it simple

${ }^{5}$ When no label is defined then local name is taken 


$$
T F\left(t_{i j}\right)=\sum_{k=1}^{n} w_{k}
$$

Final weight is marked as term frequency (TF) of the term $t_{i j}{ }^{6}$ in Eq. 2 while $w_{k}$ are partial weights aggregated across the whole collection of $n$ vectors. This aggregation that has been made then leaves us with vectors of terms ordered by their importance regarding the domain.

Term frequency (or in our case term importance) has been proven useful within information retrieval mainly because of its simplicity [7]. Despite the term frequency being an enhancement of term-weighting, the use in isolation cannot ensure an acceptable retrieval performance. Specifically, when the high frequency terms are not concentrated in a few particular documents, but they are instead distributed across the whole collection, all documents tend to be retrieved - and this severely affects the search precision. Here is where inverse document frequency comes along to help to suppress negative aspects of using term frequency solely. The reason why IDF has not been adopted into our technique is that retrieving term frequency in the rest of the corpus causes further delays what results in the decrease of practicality of the proposed approach. What we did though was making a use of vectors which we have accessible already (typically user compares an ontology against several similar domains for which the description vector was generated and these are accessible) and compute inverse document frequency out of them, considering occurrences of tested terms in other vectors while these vectors play the role of corpus. In other words: the $I D F$ factor varies inversely with the number of vectors $d f_{i j}$ to which a term is assigned and collection of $\mathrm{N}$ vectors.

$$
I D F\left(t_{i j}\right)=\log _{10} \frac{N}{d f_{i j}}
$$

Final weight is therefore computed by using Eq.2 and 3:

$$
w_{i j}=T F\left(t_{i j}\right) I D F\left(t_{i j}\right)
$$

After computing weights for all terms from all different domains we can build the Vector space model:

$F(N \times M)=\left(\begin{array}{c}d_{1} \\ d_{2} \\ \vdots \\ d_{N}\end{array}\right)=\left(\begin{array}{cccc}w_{1,1} & w_{1,2} & \cdots & w_{1, M} \\ w_{2,1} & w_{2,2} & \cdots & w_{2, M} \\ \vdots & \vdots & \ddots & \vdots \\ w_{N, 1} & w_{N, 2} & \cdots & w_{N, M}\end{array}\right)$

Vector space model contains vector description of $N$ domains while every domain is defined by its vector description of the length $M$.

\section{Key concepts summary}

Vector description discussed in the previous section serves as a reference model of the domains against which ontologies may be compared and found the best match between

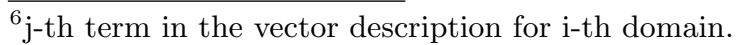

the ontology and a domain. When comparing two data resources it is crucial for them to be in the same form which is not fulfilled in this case. The ontology is represented by a complex graph that cannot be directly compared to the vector. The problem was resolved by transforming the ontology into vectors to resemble domains vector description.

The transition from the graph to a set of vectors is made in two steps:

\section{Key concepts identification \\ 2. Vector description of the key concepts}

Whereas the former deals with reducing the total number of concepts in ontology and replacing them with only those with the highest information value, the latter extends the definition of found key concepts by building vectors from their context.

\subsection{Key concepts identification}

The work in this paper is associated with the approach taken by Peroni [2] that is based on identifying so-called key concepts in an ontology, to generate a meaningful snapshot of an ontology and facilitate the process of ontology understanding. The notion of key concepts is highly subjective, but one easily follows the rationale of choosing $n$ best descriptions of the content of a particular ontology. The motivation of key concepts identification was, to some extent, to reflect the role of so-called natural categories of the cognitive science.

While specific details on transition from the abstract notion of natural categories to specific ontology measures can be found in [2], we summarize the main aspects used in our visual extension of the original algorithm. The two core cognitive measures in choosing key descriptions for an ontology are:

concept centrality is a common measure e.g. in social network analysis, where it indicates how many times a given node appears in all paths between the root and leaves.

label simplicity reflects the number of compound terms forming the concept name.

While the algorithm maximizes the former measure, the latter measure is minimized (preferring simpler names to more complicated ones). Applying these measures leave us with concepts located in the middle layer of taxonomy as they are more expressive, in terms of user understanding. General concepts at the top of taxonomy, having covered almost all the other concepts in the ontology, are often too general and have several meanings. On the other side of the scale are specific concepts located at the bottom of the taxonomy which are so specific that are unknown to a non-expert user.

\subsection{Vector description of key concepts}

Key concepts on their own are a good approximation of the ontology content, they form essentially a list. One can then choose a list with five, ten or more items to reflect the breadh and granularity of coverage. In order to move 


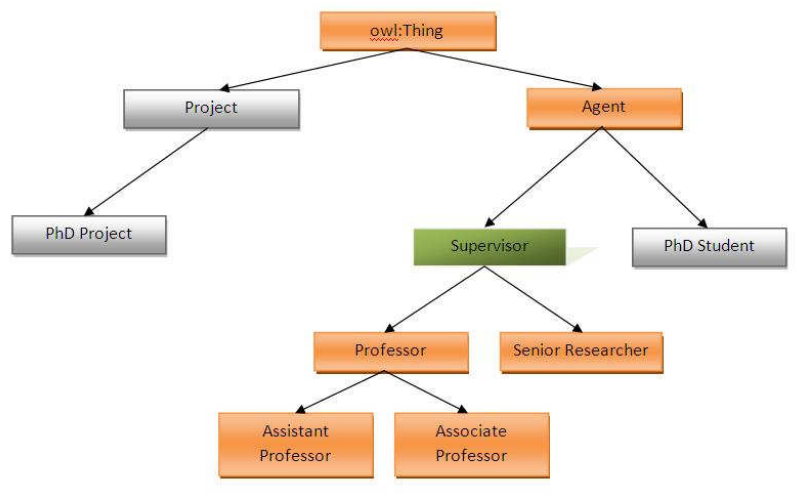

Figure 3: Graph of the evaluated ontology. Key concept in green square and all concepts considered when building vector description in orange blocks.

to proper ontology summaries we add to the labels appropriate topological data and context. The introduction of topology is essentially about linking the currently selected key concepts taking into account the actual is $A$ hierarchy of the visualized ontology. Three types of connections are calculated to cover context:

1. direct serialization (isA or subClassOf)

2. direct instantiation (typeOf)

3. distant ancestor

Whilst first two types are self-explanatory, the last one needs to be specified. The distant ancestor to a key concept is such a concept that has no direct connection with the key concept but an indirect path exists. Its simple definition is: $C_{0}<i s A>C_{1}<i s A>C_{2}<i s A>C_{3}$ exists in a given ontology, possibly within maximum $k$ number of hops, where $C_{3}$ is key concept. In that particular case, the distant ancestor is considered $C_{1}$ regarding the key concept $C_{3}$. This extension against domain vector description was done to extend the size of the final vector description of a key concept.

The rest of the key concept vector description calculation performs according to the same pattern as vector description of the domain. The only difference is that while the domain vector description is aggregated from several partial vectors, the key concept description has only one vector as there is only one ontology.

\section{Similarity measures of vector descriptions and their visualization}

In order to establish semantic similarity of two elements, we need to have more than just their names. Technique employed to collect and store information about domains and/or key concepts were discussed in the previous sections and once the necessary background calculations are done we can make a use of these data.

The reason for creating vector descriptions for domains and key concepts is to create homogeneous resources which may be compared mutually, resulting in establishing semantic similarity for a pair (key concept - domain). The similarity (so-called cosine similarity) is calculated by the formula:

$$
S_{k}=\frac{\sum_{l=1}^{m} x_{i l} x_{j l}}{\sqrt{\sum_{l=1}^{m} x_{i l}^{2} \sum_{l=1}^{m} x_{j l}^{2}}}
$$

After performing the calculation for every pair (key concept - domain) we obtain similarities $S\left(x_{i}, x_{j}\right)$ where $x_{i}$ is the vector of $\mathrm{i}$-th key concept and $x_{j}$ is the vector of $\mathrm{j}$-th domain. Similarity matrix is then defined as:

$$
S=\left(\begin{array}{ccc}
S_{11} & \ldots & S_{1 M} \\
\vdots & \ddots & \vdots \\
S_{N 1} & \ldots & S_{N M}
\end{array}\right)
$$

The similarity matrix is subsequently used to determine the best fit for every key concept which is used to create topical map out of domains ${ }^{7}$ and key concepts ${ }^{8}$.

On the Fig. 4 there is a screenshot of proposed visualization technique implemented as a plug-in for $\mathrm{NeOn}$ toolkit. The picture consists of two elements which are background and an ontology being visualized. The background serves mostly as a definition of user interest, what the user is actually looking for, while coming across various ontologies. Thus background is a reference environment that helps the user to assess or interpret various parts of an ontology and as such defines the appearance of the visualised ontology. The yellow circles on the other hand represent the ontology as a set of concepts with their mutual connections. To avoid overwhelming presentation only key concepts are depicted. These therefore are concepts with the highest significance and, to some extend, replace all other concepts in the ontology.

The key concepts do not entirely replace the other concepts but are used as primary visual elements rather than using root on abstract level of taxonomy (such as owl:Thing). In this way we are presenting to users more data without actually increasing the screen estate or diverging from the nature of visualized information.

Another innovation that is implemented in our summarydriven visualization is inspired by the notion of term (tag) popularity that is pretty familiar from the Web 2.0 applications. The original idea refers to terms in a respective tag cloud and often suggests that tags in smaller fonts are less important. Nonetheless, the idea of associating some ontological measure with the size of the visualized concepts has proven to be reusable and therefore used in our technique.

In our case we associate the sizing of key concepts with the abstract notion of conceptual zooming. Conceptual zooming can be easily explained using the map metaphor mentioned earlier. The main principle is that the amount of data (e.g. cities) shown in the map depends on the

\footnotetext{
${ }^{7}$ on the background of the map

${ }^{8}$ representing points on the map
} 


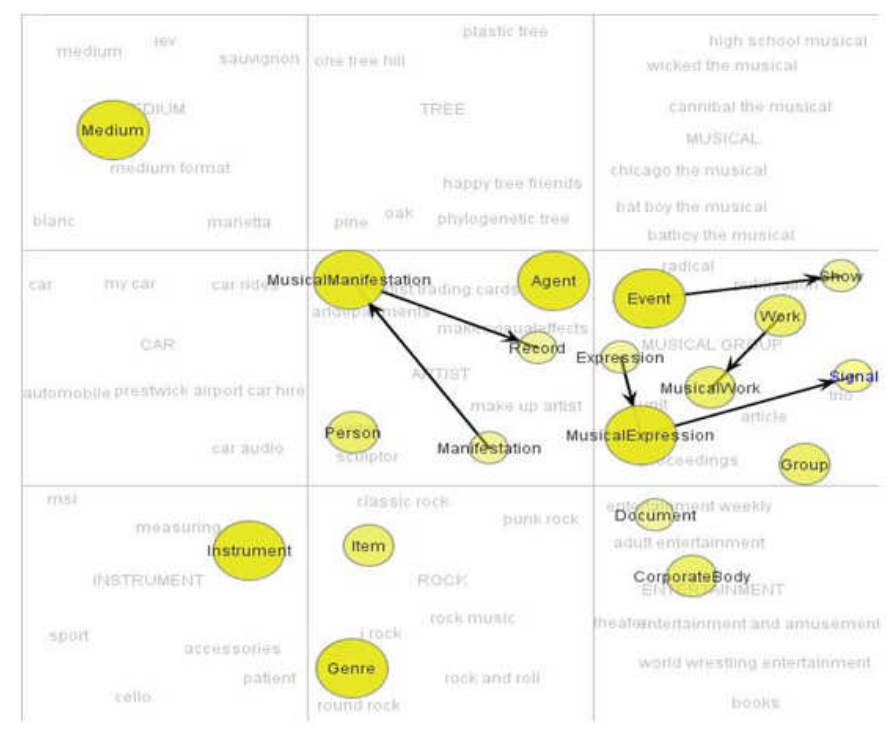

Figure 4: Screenshot of the visualization based on summaries. The domains of interest at the background define an environment in which an ontology (in this case the musicontology.owl) is depicted.

zoom level and that in turn corresponds to the map scale. The key concepts take advantage of the subjective nature of how much does one need to describe an ontology. Since the concepts that are shown among the top ten are also shown in top twenty, one can consider them more important in sense of being more likely to be used by human in summarizing what a given ontology is about.

In our extension we thus emulate key concept importance by the information value it adds to the ontology summary. Once we calculate the importance for each concept per se, one can then introduce an action of conceptual zooming or zooming at the conceptual level i.e. adding or removing key concepts (and the related direct or distant links) to the ontology summary. And all this in addition to the usual zoom of showing closer details of an image.

\section{Experimental analysis}

Discussed points of the developed visualization technique were sustained by large scale study [4] which identified a number of key points where existing visualization techniques obtained negative comments from non-experts. We aimed to address all the concerns (highlighted in the fist section) and make sure that proposed approach meets the user's demands.

\subsection{Methodology and basic conditions}

The described functionalities were embedded in the $\mathrm{NeOn}$ toolkit that gave us a chance to evaluate this approach with users. Evaluations were carried out with 5 ontology experts each of which had a brief introduction to the tool followed by a trial of all the functionalities on one ontology. Members of the group were chosen to have good grasp of existing visualization tools and therefore be able to compare them in terms of usability.

The ontology used during evaluation was picked bearing in mind its quality, scale and topic. All three parameters affect appraisal of experts and balance among these parameters impacts results heavily. With regard of comprehensibility, musiontology.rdf (for the music domain being generally familiar) was chosen. The used ontology, cre- ated in RDF specification, contains:

- 1696 triplets (statements),

- 83 classes,

- 119 properties,

- 14 instances.

The maximum depth of ontology is 5 whereas the average depth is 2-3 classes. The picture Fig. 4 shows the musicontology.rdf as presented to experts in the experiment.

\subsection{Results}

Experts stressed the feature of depicting key concepts on the map where the user can easily see the coverage for each segment of the map. This is particularly interesting when developing an ontology to identify the bits of ontology which need a further development.

Map of terms shown as the background evoke semantic distance within a scope ${ }^{9}$ although semantic distance is implemented on the level of relationship key concept background ${ }^{10}$.

Negative comments were made on the process of adding key concepts on the map, when the position is constantly changing to ensure good visibility all key concepts. Experts claim it to be confusing to users, making an impression that key concepts change their assessment.

Along the new method of depicting an ontology, our tool provides also more traditional approach of tree view visualization, ordering key concepts into hierarchical layers. This feature was considered as useful though they proposed the indented list to be more appropriate in its full length. This would be in contradiction with the aim to

\footnotetext{
${ }^{9}$ Distance on the map does not reflect similarity in meaning and terms are within a scope distributed randomly

${ }^{10}$ not key concept - key concept
} 
alleviate cognitive load of users, let alone the fact that the same approach was taken by Protege[6] developers.

Similarly, some experts do not find the grid useful. The grid works, in some extend, as a splitter which unambiguously determines borders of each scope and consequently asses key concepts to scopes.

\section{Conclusion}

In the paper we presented motivation, rationale and explanation of several user interaction capabilities embedded in our visualization technique for visualizing ontologies based on their conceptual summaries. The core of the presented approach is in working with ontology summaries instead of top-down hierarchical trees (or forests). To this extend an algorithmic suite for identifying key concepts was developed and extended by topological and navigational summaries.

The different way of facilitating the process of ontology understanding, as laid out in paper, simplifies the process of ontology understanding and makes a counterpart to traditional methods which were proven ineffective. The implemented visualization tool, underlying the proposed approach, relies on and takes advantage of several thirdparty products:

Watson necessary for a part of the functionality of the key concept algorithm as well as vector description of the domain, where Watson's index acts as an ontology corpus. Watson also covers the access to the ontology corpus, whereby we gather information either about key concept ${ }^{11}$ or domain ${ }^{12}$.

Graph visualization is built on top of the $J U N G$ (Java Universal Network/Graph library), which is an Open Source product supporting basic graph models.

Other libraries comprise a range of previously developed software modules supporting XML parsing, work with taxonomies, etc.

While testing the implemented prototype, a set of drawbacks has been spotted which influence in negative way the final results of the algorithm. Due to the inequality of distribution of ontologies in the used corpus, in terms of covering the domains, we encountered a lack of quality ontologies in some domains. The quality of the corpus towards particular domain is crucial as having a significant effect on the quality of vector description for the domain. Important point to raise is that if in ontologies are favoured terms that are general, the specific concepts will be excluded from the vector and consequently from the comparison process.

Another in-built issue we became aware of when scaling the test is the latency for extra popular ${ }^{13}$ domains. This problem was resolved via a cache scheme, storing vectors calculated in the past.

Next limitation is due to low-quality ontologies indexed in an ontology corpus. These ontologies are either automat-

\footnotetext{
${ }^{11}$ when building vector description of the key concept

${ }^{12}$ while describing domain by vector description

${ }^{13}$ popularity is measured by the number of ontologies which describe the domain
}

ically generated or are created without following common practice. Since these are numerous, they are likely to occur in the vector description. We partially solve the problem by applying stop words reduction in the vector together with a heuristic algorithm to the reduce number of elements in the vector with no information value.

The motivation for the future research should be therefore to improve the quality of vector description process what creates a potential for further improvements not only in the field of ontology visualization but also as a general representation of the domain that can be used e.g. in classification. From the perspective of ontology visualization, the next step should be to extend the technique to visualize more than one ontology at the same time which allows to see how different ontologies complement/overlap.

Acknowledgements. The work presented in this paper was supported by the Slovak Grant Agency of Ministry of Education and Academy of Science of the Slovak Republic within the $1 / 0042 / 10$ project 'Methods for identification, annotation, search, access and composition of services using semantic metadata in support of selected process types'.

\section{References}

[1] M. d'Aquin, C. Baldassare, L. Gridinoc, S. Angeletou, M. Sabou, and E. Motta. Watson: A gateway for next generation semantic web applications. Poster session of the Internatinal Semantic Web Conference, ISWC, 2007.

[2] M. d'Aquin, E. Motta, and S. Peroni. Identifying key concepts in an ontology, through the integration of cognitive principles with statistical and topological measures. Knowledge Media Institute, 2006.

[3] L. Ding. Swoogle: A search and metadata engine for the semantic web. In Proceedings of the Thirteenth ACM Conference on Information and Knowledge Management, November 2004.

[4] M. Dzbor, E. Motta, C. Builarabda, J. Gomez-Perez, O. Goerlitz, and $\mathrm{H}$. Lewen. Analysis of user needs, behaviours and requirements wrt interfaces and navigation of ontologies. Deliverable report, D(4.1.1):5-14, August 2006.

[5] A. Katifori, C. Halatsis, G. Lepouras, C. Vassilakis, and E. Giannopoulou. Ontology visualization methods - a survey. ACM Comput. Surv., 39(4):43, October 2007.

[6] N. Noy, W. R. Fergason, and A. M. Musen. The knowledge model of protege. In Proceedings of the 2nd International Conference on Knowledge Engineering and Knowledge Management, 2000.

[7] G. Salton and C. Buckley. Term-Weighting Approaches in Aoutomatic Text Retrieval. 1988.

[8] J. Vrana. Conceptual visualization of ontological models. Technical University in Kosice, Kosice, 2010.

\section{Selected Papers by the Author}

J. Vrana, M. Mach. Key concepts extended by vector desriptions to interpret the meaning of ontologies. Acta Electrotechnica et Informatica. (submitted), 2010.

J. Vrana, K. Machova, M. Dzbor. Ontologies evaluation and visualization. Znalosti 2009, Brno, Ceska republika. str. 333-336.

J. Vrana, K. Machova, M. Dzbor. Podpora semantickeho vyhladavania na webe. Znalosti 2008, Bratislava, Slovensko. str. 399-402. 